This item was submitted to Loughborough's Research Repository by the author.

Items in Figshare are protected by copyright, with all rights reserved, unless otherwise indicated.

\title{
Bridge health monitoring system based on vibration measurements
}

PLEASE CITE THE PUBLISHED VERSION

http://dx.doi.org/10.1007/s10518-008-9067-4

PUBLISHER

(C) Springer Verlag

VERSION

AM (Accepted Manuscript)

LICENCE

CC BY-NC-ND 4.0

REPOSITORY RECORD

Ntotsios, Evangelos, Costas Papadimitriou, Panagiotis Panetsos, Grigorios Karaiskos, Kyriakos Perros, and Philip C. Perdikaris. 2019. "Bridge Health Monitoring System Based on Vibration Measurements". figshare. https://hdl.handle.net/2134/10762. 
This item was submitted to Loughborough's Institutional Repository (https://dspace.lboro.ac.uk/) by the author and is made available under the following Creative Commons Licence conditions.

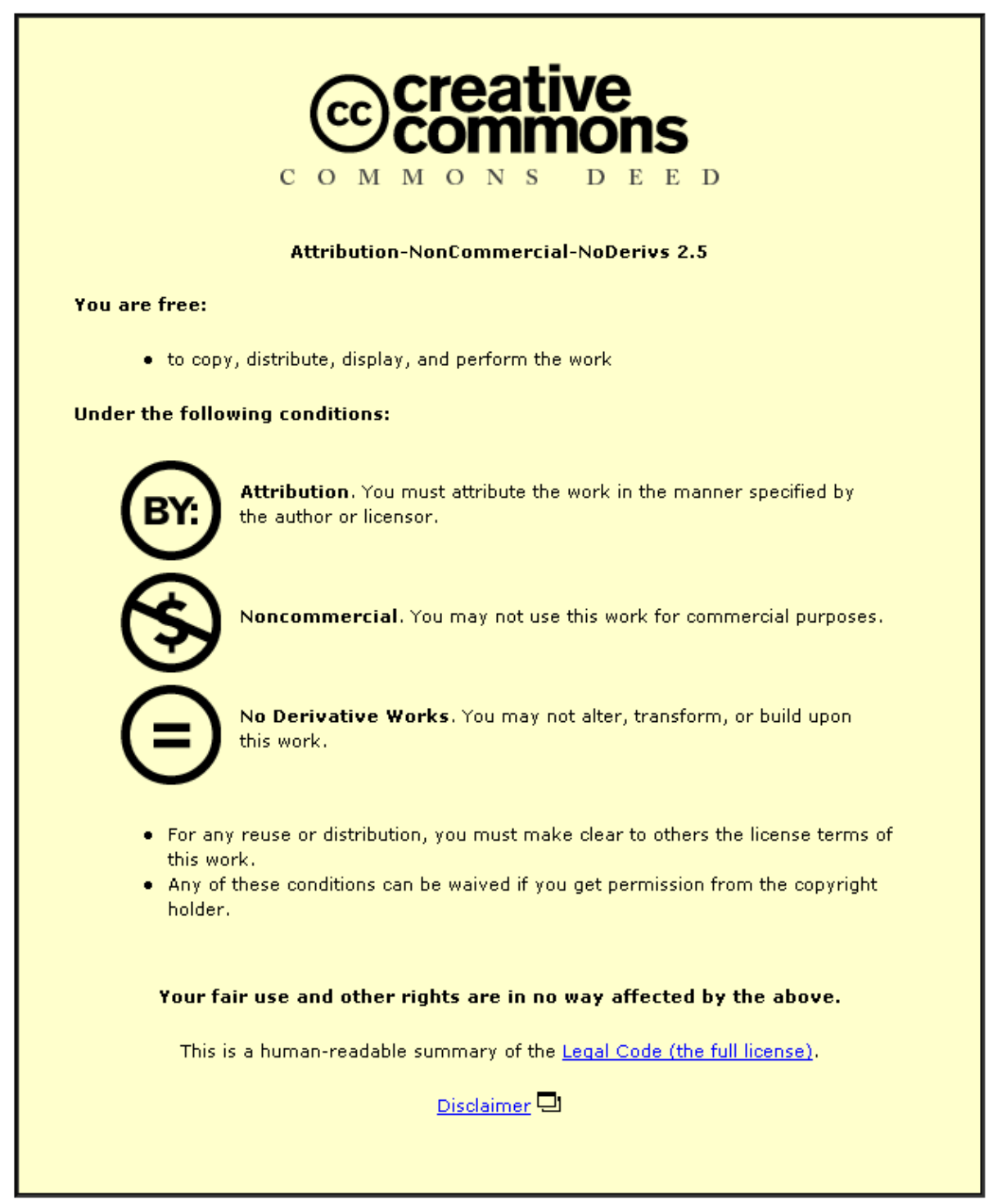

For the full text of this licence, please go to: http://creativecommons.org/licenses/by-nc-nd/2.5/ 


\title{
Bridge health monitoring system based on vibration measurements
}

\author{
Evaggelos Ntotsios ${ }^{1}$, Costas Papadimitriou ${ }^{2}$, Panagiotis Panetsos ${ }^{3}$, \\ Grigorios Karaiskos ${ }^{1}$, Kyriakos Perros ${ }^{1}$, Philip C. Perdikaris ${ }^{4}$
}

\begin{abstract}
A bridge health monitoring system is presented based on vibration measurements collected from a network of acceleration sensors. Sophisticated structural identification methods, combining information from the sensor network with the theoretical information built into a finite element model for simulating bridge behaviour, are incorporated into the system in order to monitor structural condition, track structural changes and identify the location, type and extent of damage. This work starts with a brief overview of the modal and model identification algorithms and software incorporated into the monitoring system and then presents details on a Bayesian inference framework for the identification of the location and the severity of damage using measured modal characteristics. The methodology for damage detection combines the information contained in a set of measurement modal data with the information provided by a family of competitive, parameterized, finite element model classes simulating plausible damage scenarios in the structure. The effectiveness of the damage detection algorithm is demonstrated and validated using simulated modal data from an instrumented R/C bridge of the Egnatia Odos motorway, as well as using experimental vibration data from a laboratory small-scaled bridge section.
\end{abstract}

Keywords: Structural health monitoring, Model updating, Bayesian inference, Structural identification, Damage detection.

\footnotetext{
${ }^{1}$ Graduate Student, Department of Mechanical and Industrial Engineering, University of Thessaly, Volos 38334, Greece (e-mail: entotsio@uth.gr, gkaraiskos@uth.gr, kyperros@uth.gr)

${ }^{2}$ Professor, Department of Mechanical and Industrial Engineering, University of Thessaly, Volos 38334, Greece (e-mail: costasp@uth.gr)

${ }^{3}$ Civil Engineer, PhD, Egnatia Odos S.A., Capital Maintenance Department, 6th km Thessaloniki-Thermi, P.O. Box 30, GR 57001 Thermi, Greece (e-mail: ppane@egnatia.gr)

${ }^{4}$ Professor, Department of Civil Engineering, University of Thessaly, Volos 38334, Greece (e-mail: filperd@uth.gr)
} 


\section{Introduction}

Successful health monitoring of structural systems depends to a large extent on the integration of cost-effective intelligent sensing techniques, accurate physicsbased computational models simulating structural behavior, effective system identification methods, sophisticated health diagnosis algorithms, as well as decision-making expert systems to guide management in planning optimal costeffective strategies for system maintenance, inspection and repair/replacement. Structural integrity assessment of highway bridges can in principle be accomplished using continuous structural monitoring based on vibration measurements. Taking advantage of modern technological capabilities, vibration data can be obtained remotely, allowing for a near real-time assessment of the bridge condition. Using these measurements, it is possible to identify the dynamic modal characteristics of the bridge and update a theoretical finite element model. The results from the identification and updating procedures are useful to examine structural integrity after severe loading events (strong winds and earthquakes), as well as bridge condition deterioration due to long-term corrosion, fatigue and water scouring.

Algorithms and graphical user interface (GUI) software have been developed for monitoring the bridges of the Egnatia Odos highway system. The bridge structural health monitoring system combines information from finite element structural models representing the behavior of bridges and vibration measurements recorded using an array of sensors. It incorporates algorithms related to (1) optimal experimental design, (2) experimental modal analysis from ambient and earthquake-induced vibrations, (3) finite element model updating, and (4) structural damage detection based on finite element model updating.

Optimal experimental design methods refer to algorithms for optimizing the location and number of sensors in the structure such that the measure data contain the most important information for structural identification purposes. Algorithms based on information theory and using a nominal finite element model of the structure, have been proposed to address this problem (Kirkegaard and Brincker 1994; Papadimitriou 2005). Effective heuristic optimization tools have also been developed and implemented into software for efficiently solving the resulting nonlinear single- and multi-objective optimization problems involving discrete- 
valued variables. It has been demonstrated that optimal sensor configurations depend on several factors, including the purpose of the analysis (modal analysis, model updating or damage detection), parameterization schemes used in model updating, probable damage scenarios that are monitored, as well as the type and number of modes identified from the data.

Experimental modal analysis algorithms for bridge structures process either ambient or earthquake-induced vibrations in order to identify the modal characteristics. A brief overview with references of modal identification methods was given in the companion paper (Ntotsios et al. 2008). Recent efforts have been concentrated on developing algorithms and graphical user interface (GUI) software for automated modal analysis based on ambient vibrations with minimum user interference (e.g. Goursat et al. 2000; Verboven et al. 2004; Peeters et al 1999, Reynders and De Roeck 2007). As part of the proposed bridge monitoring system, GUI software has been developed from the University of Thessaly group for computing the modal properties of bridges by processing either ambient or earthquake acceleration recordings (Ntotsios et al 2008).

Finite element model updating methods based on modal data are often used to develop high fidelity models so that model predictions are consistent with measured data. The need for model updating arises because there are always assumptions and numerical errors associated with the process of constructing a theoretical model of a structure and predicting its response using the underlined model. Reviews of model updating methodologies based on modal data can be found in the work by Mottershead and Friswell (1993). Moreover, model updating methodologies are useful in predicting the structural damage by continually updating the structural model using vibration data (Hemez and Farhat 1995; Sohn and Law 1997; Fritzen et al. 1998; Doebling et al. 1998; Vanik et al. 2000; Papadimitriou 2004; Lam et al. 2004; Teughels and De Roeck 2005; Lam et al. 2007). Such updated models obtained periodically throughout the lifetime of the structure can be further used to update the response predictions and lifetime structural reliability based on available data (Papadimitriou et al. 2001). Graphical user interface software has been developed from the University of Thessaly group as part of the bridge monitoring system for automating the model updating process using various modal-based model updating methodologies (Christodoulou and Papadimitriou 2007). The software interfaces with the commercial COMSOL 
Multiphysics (COMSOL AB 2005) software that provides the necessary finite element modeling tools.

This work presents details for the damage detection algorithm used in the monitoring system. The damage detection algorithm is based on reconciling finite element models with data collected before and after damage using a Bayesian methodology (Yuen 2002; Beck and Yuen 2004; Papadimitriou and Katafygiotis 2004) for selecting a model class from a family of competitive parameterized model classes. The Bayesian methodology is outlined in Section 2, based on measured modal characteristics. The structural damage identification, outlined in Section 3, is accomplished by associating each parameterized model class in the family to a damage pattern in the structure, indicative of the location of damage. Using the Bayesian model selection framework, the probable damage locations are ranked according to the posterior probabilities of the corresponding model classes. The severity of damage is then inferred from the posterior probability of the model parameters derived for the most probable model class. Based on asymptotic approximations, the damage diagnosis involves solving a series of model updating problems for each model class in the family. Examples illustrating the applicability of the proposed method are presented in Section 4 using simulated modal data for the Polymylos bridge, as well as measured data from a laboratory small-scale section of a bridge.

\section{Bayesian method for finite element model class selection}

Let $D=\left\{\hat{\omega}_{r}, \hat{\phi}_{r} \in R^{N_{0}}, r=1, \cdots, m\right\}$ be the available measured data consisting of modal frequencies $\hat{\omega}_{r}$ and modeshape components $\hat{\phi}_{r}$ at $N_{0}$ measured DOFs, where $m$ is the number of observed modes. Consider a family of $\mu$ alternative, competing, parameterized finite element model classes, designated by $M_{i}$, $i=1, \cdots, \mu$, and let $\boldsymbol{\theta}_{i} \in R^{N_{\theta_{i}}}$ be the free parameters of the model class $M_{i}$, where $N_{\theta_{i}}$ is the number of parameters in the set $\boldsymbol{\theta}_{i}$. Let $\Pi\left(\boldsymbol{\theta}_{i} ; M_{i}\right)=\left\{\omega_{r}\left(\boldsymbol{\theta}_{i} ; M_{i}\right), \boldsymbol{\phi}_{r}\left(\boldsymbol{\theta}_{i} ; M_{i}\right) \in R^{N_{0}}, r=1, \cdots, m\right\}$ be the predictions of the modal frequencies and modeshapes from a particular model in the model class $M_{i}$ corresponding to a particular value of the parameter set $\boldsymbol{\theta}_{i}$. 
A Bayesian probabilistic framework is next briefly presented which is attractive to address the problem of comparing two or more competing model classes and selecting the optimal model class based on the available data. The Bayesian approach to statistical modeling uses probability as a way of quantifying the plausibilities associated with the various model classes $M_{i}$ and the parameters $\boldsymbol{\theta}_{i}$ of these model classes given the observed data $D$. Before the selection of data, each model class $M_{i}$ is assigned a probability $P\left(M_{i}\right)$ of being the appropriate class of models for modeling the structural behavior. Using Bayes' theorem, the posterior probabilities $P\left(\mathrm{M}_{i} \mid D\right)$ of the various model classes given the data $D$ is

$$
P\left(\mathrm{M}_{i} \mid D\right)=\frac{p\left(D \mid \mathrm{M}_{i}\right) P\left(\mathrm{M}_{i}\right)}{d}
$$

where $d$ is selected so that the sum of all model probabilities equals to one, and $p\left(D \mid M_{i}\right)$ is the probability of observing the data from the model class $M_{i}$, given by

$$
p\left(D \mid \mathbf{M}_{i}\right)=\int_{\Theta_{i}} p\left(D \mid \boldsymbol{\theta}_{i}, \boldsymbol{\sigma}_{i}, M_{i}\right) \pi\left(\boldsymbol{\theta}_{i}, \boldsymbol{\sigma}_{i} \mid \mathbf{M}_{i}\right) d \boldsymbol{\sigma}_{i} d \boldsymbol{\theta}_{i}
$$

where $\Theta_{i}=\left\{\boldsymbol{\theta}_{i}: \mathbf{0}<\boldsymbol{\theta}_{i} \leq \boldsymbol{\theta}_{i}^{u}\right\}$ is the domain of integration in (2) that depends on the range of variation of the parameter set $\boldsymbol{\theta}_{i}$, and $\boldsymbol{\theta}_{i}^{u}$ are the values of $\boldsymbol{\theta}_{i}$ at the undamaged condition of the structure. In (2), $p\left(D \mid \boldsymbol{\theta}_{i}, \boldsymbol{\sigma}_{i}, M_{i}\right)$ is the likelihood of observing the data from a given model in the model class $M_{i}$. This likelihood is obtained using predictions $\Pi\left(\boldsymbol{\theta}_{i} ; M_{i}\right)$ from the model class $M_{i}$ and the associated probability models for the vector of prediction errors $\boldsymbol{e}^{(i)}=\left[e_{1}^{(i)}, \cdots, e_{m}^{(i)}\right]$ defined as the difference between the measured modal properties involved in $D$ for all modes $r=1, \cdots, m$ and the corresponding modal properties predicted by a model in the model class $M_{i}$. Specifically, the model error $\boldsymbol{e}_{r}^{(i)}=\left[e_{\omega_{r}}^{(i)} \boldsymbol{e}_{\phi_{r}}^{(i)}\right]$ for the model class $M_{i}$ is given separately for the modal frequencies and modeshapes from the prediction error equations:

$$
\hat{\omega}_{r}=\omega_{r}\left(\boldsymbol{\theta}_{i} ; M_{i}\right)+\hat{\omega}_{r} e_{\omega_{r}}^{(i)} \quad r=1, \ldots, m
$$




$$
\hat{\boldsymbol{\phi}}_{r}=\beta_{r}^{(i)} \boldsymbol{\phi}_{r}\left(\boldsymbol{\theta}_{i} ; M_{i}\right)+\left\|\hat{\phi}_{r}\right\| \boldsymbol{e}_{\phi_{r}}^{(i)} \quad r=1, \ldots, m
$$

where $\beta_{r}^{(i)}=\hat{\phi}_{r}^{T} \phi_{r}^{(i)} / \phi_{r}^{(i) T} \phi_{r}^{(i)}$, with $\phi_{r}^{(i)} \equiv \phi_{r}\left(\boldsymbol{\theta}_{i} ; M_{i}\right)$, is a normalization constant that accounts for the different scaling between the measured and the predicted modeshape. The model prediction errors are due to modeling error and measurement noise. Herein, they are modeled as independent Gaussian zero-mean random variables with variance $\boldsymbol{\sigma}_{i}^{2}$. Also, given the model class $M_{i}$, the prior probability distribution $\pi\left(\boldsymbol{\theta}_{i}, \boldsymbol{\sigma}_{i} \mid M_{i}\right)$, involved in (2), of the model and the prediction error parameters $\left[\boldsymbol{\theta}_{i}, \boldsymbol{\sigma}_{i}\right]$ of the model class $M_{i}$ are assumed to be independent and of the form $\pi\left(\boldsymbol{\theta}_{i}, \boldsymbol{\sigma}_{i} \mid \mathrm{M}\right)=\pi_{\theta}\left(\boldsymbol{\theta}_{i}\right) \pi_{\sigma}\left(\boldsymbol{\sigma}_{i}\right)$.

Under the assumption that the prior distributions $\pi_{\theta}\left(\boldsymbol{\theta}_{i}\right)$ are non-informative uniform distributions over the range of variation of $\boldsymbol{\theta}_{i}$, and using asymptotic approximations valid for large number of data to approximate the integral (2), the probability of the model class $M_{i}$ is given by (Papadimitriou and Katafygiotis 2004)

$$
\log P\left(\mathbf{M}_{i} \mid D\right)=-N_{J} \log \left[J\left(\hat{\boldsymbol{\theta}}_{i} ; \mathbf{M}_{i}, D\right)\right]+\beta\left(\hat{\boldsymbol{\theta}}_{i} ; \mathbf{M}_{i}, D\right)+P\left(\mathbf{M}_{i}\right)+d
$$

where

$$
J\left(\boldsymbol{\theta}_{i} ; M_{i}, D\right)=\frac{1}{m} \sum_{r=1}^{m}\left[\frac{\omega_{r}\left(\boldsymbol{\theta}_{i} ; M_{i}\right)-\hat{\omega}_{r}}{\hat{\omega}_{r}}\right]^{2}+\frac{1}{m} \sum_{r=1}^{m} \frac{\left\|\alpha_{r} \boldsymbol{\phi}_{r}\left(\boldsymbol{\theta}_{i} ; \mathbf{M}_{i}\right)-\hat{\boldsymbol{\phi}_{r}}\right\|^{2}}{\|\hat{\boldsymbol{\phi}}\|^{2}}
$$

represents the measure of fit between the measured modal data and the modal data predicted by a particular model in the class $M_{i},\|\cdot\|$ is the usual Euclidian norm, $\hat{\boldsymbol{\theta}}_{i}$ is the value that minimizes the measure of fit $J\left(\boldsymbol{\theta}_{i} ; M_{i}, D\right)$ in (6), $d$ is constant independent of the model class $M_{i}, N_{J}=\left(m N_{0}-1\right) / 2$, and the factor $\beta\left(\hat{\boldsymbol{\theta}}_{i} ; \mathrm{M}_{i}, D\right)$ in (5), known as the Ockham factor, simplifies for large number of data $N_{J}$ to (Yuen 2002, Beck and Yuen 2004)

$$
\beta\left(\hat{\boldsymbol{\theta}}_{i} ; \mathrm{M}_{i}, D\right)=\beta_{i}=-\frac{N_{\theta_{i}}}{2} \log N_{J}
$$

where it is evident that it depends from the number $N_{\theta_{i}}$ of the model parameters involved in the model class $M_{i}$. It should be pointed out that the optimisation 
problem for finding $\hat{\boldsymbol{\theta}}_{i}$ for each model class are solved using efficient hybrid optimization techniques that guarantee the estimation of the global optimum (Christodoulou and Papadimitriou 2007).

It should be noted that the asymptotic approximation is valid if the optimal value $\hat{\boldsymbol{\theta}_{i}}$ belongs to the domain $\Theta_{i}$ of integration in (2). For the cases for which this condition is violated or for the case for which more accurate estimates of the integral are required, one can use alternative stochastic simulation methods to evaluate the integral (2). Specifically, Monte Carlo simulation can be used which may require a large number of samples and can be computationally inefficient. Importance sampling methods (Papadimitriou et al. 1997) as well as Markov Chain Monte Carlo (Au 2001; Beck and Au 2002; Katafygiotis and Cheung 2002) may be applicable to efficiently evaluate the integral in (2).

The optimal model class $M_{\text {best }}$ is selected as the model class that maximizes the probability $P\left(M_{i} \mid D\right)$ given by (5). It is evident that the selection of the optimal model class depends on the measure of fit $J\left(\hat{\boldsymbol{\theta}}_{i} ; \mathrm{M}_{i}, D\right)$ between the measured modal characteristics and the modal characteristics predicted by the optimal model of a model class $M_{i}$. Thus, the first term in (5) gives the dependence of the probability of a model class $M_{i}$ from how well the model class predicts the measurements. The smaller the value of $J\left(\hat{\boldsymbol{\theta}}_{i} ; \mathrm{M}_{i}, D\right)$, the higher the probability $P\left(M_{i} \mid D\right)$ of the model class $M_{i}$. Based on the Ockham factor $\beta_{i}$ simplified in (7), the ordering of the model classes in (5) also depends on the number $N_{\theta_{i}}$ of the structural model parameters that are involved in each model class. Specifically, model classes with large number of parameters are penalized in the selection of the optimal model class.

Finally, the probability distribution $p\left(\boldsymbol{\theta}_{i} \mid D, \mathrm{M}_{i}\right)$ quantifying the uncertainty in the parameters $\boldsymbol{\theta}_{i}$ of a model class $M_{i}$ given the data is obtained by applying Bayes' theorem (Beck and Katafygiotis 1998) and then finding the marginal distribution of the structural model parameters. For the model class $M_{i}$, this yields (Katafygiotis et al. 1998)

$$
p\left(\boldsymbol{\theta}_{i} \mid D, M_{i}\right)=c_{i} J\left(\boldsymbol{\theta}_{i} ; M_{i}, D\right)^{-N_{J}} \pi_{\theta}\left(\boldsymbol{\theta}_{i}\right)
$$


where $c_{i}$ is a normalizing constant guaranteeing that the PDF integrates to one. It is evident from (8) that the most probable model that maximizes the probability distribution $p\left(\boldsymbol{\theta}_{i} \mid D, \mathrm{M}_{i}\right)$ of the structural parameters of the model class $\mathrm{M}_{i}$ is the $\hat{\boldsymbol{\theta}}_{i}$ that also minimizes the measure of fit function $J\left(\boldsymbol{\theta}_{i} ; \mathrm{M}_{i}, D\right)$ in (6) with respect to $\boldsymbol{\theta}_{i}$, provided that $\pi_{\theta}\left(\boldsymbol{\theta}_{i}\right)=\pi_{i}$ is selected to be constant. The most probable value of the parameter set that corresponds to the most probable model class $M_{\text {best }}$ is denoted by $\hat{\boldsymbol{\theta}}_{\text {best }}$.

\section{Damage detection methodology}

The Bayesian inference methodology for model class selection based on measured modal data is next applied to detect the location and severity of damage in a structure. A substructure approach is followed where it is considered that the structure is comprised of a number of substructures. It is assumed that damage in the structure causes stiffness reduction in one of the substructures. In order to identify which substructure contains the damage and predict the level of damage, a family of $\mu$ model classes $M_{1}, \cdots, M_{\mu}$ is introduced, and the damage identification is accomplished by associating each model class to damage contained within a substructure. For this, each model class $M_{i}$ is assumed to be parameterized by a number of structural model parameters $\boldsymbol{\theta}_{i}$ controlling the stiffness distribution in the substructure $i$, while all other substructures are assumed to have fixed stiffness distributions equal to those corresponding to the undamaged structure. Damage in the substructure $i$ will cause stiffness reduction which will alter the measured modal characteristics of the structure. The model class $M_{i}$ that "contains" the damaged substructure $i$ will be the most likely model class to observe the modal data since the parameter values $\boldsymbol{\theta}_{i}$ can adjust to the modified stiffness distribution of the substructure $i$, while the other modal classes that do not contain the substructure $i$ will provide a poor fit to the modal data. Thus, the model class $M_{i}$ can predict damage that occurs in the substructure $i$ and provide the best fit to the data.

Using the Bayesian model selection framework in Section 2, the model classes are ranked according to the posterior probabilities based on the modal data. The most probable model class $M_{\text {best }}$ that maximizes $p\left(M_{i} \mid D\right)$ in (5), through its 
association with a damage scenario on a specific substructure, will be indicative of the substructure that is damaged, while the most probable value $\hat{\boldsymbol{\theta}}_{\text {best }}$ of the model parameters of the corresponding most probable model class $M_{\text {best }}$, compared to the parameter values of the undamaged structure, will be indicative of the severity of damage in the identified damaged substructure. For this, the percentage change $\Delta \boldsymbol{\theta}_{i}$ between the best estimates of the model parameters $\hat{\boldsymbol{\theta}}_{i}$ of each model class and the values $\hat{\boldsymbol{\theta}}_{i, u n d}$ of the reference (undamaged) structure is used as a measure of the severity (magnitude) of damage computed by each model class $M_{i}, i=1, \ldots, \mu$.

It should be noted that a model class from the selected family that does not contain the damage may be promoted by the methodology as the best model class. This situation arises when the sensor configuration (number and locations of sensors) is inadequate to give enough information for identifying all model classes in the presence of model and measurement errors. Optimal sensor location strategies (Papadimitriou 2005) should be used to identify the number and locations of sensors that give the most informative data for reliably identifying all model classes simultaneously and minimize or avoid false detection situations.

The selection of the competitive model classes $M_{i}, i=1, \ldots, \mu$ depends on the type and number of alternative damage scenarios that are expected to occur or desired to be monitored in the structure. The $\mu$ model classes can be introduced by a user experienced with the type of structure monitored. The prior distribution $P\left(M_{i}\right)$ in (5) of each model class or associated damage scenario is selected based on the previous experience for the type of bridge that is studied. For the case where no prior information is available, the prior probabilities are assumed to be equal, $P\left(M_{i}\right)=1 / \mu$, for all introduced damage scenarios.

\section{Applications}

\subsection{Damage detection for Polymylos bridge using simulated modal data}

The effectiveness of the damage detection methodology is first validated using simulated modal data from the Polymylos bridge of the Egnatia Odos motorway. 
The description of the Polymylos bridge along with the 1350-DOF finite element model used to represent its behavior is presented in the companion paper (Ntotsios et al. 2008). Two damage cases were considered as shown schematically in Figure 1. The damages correspond to stiffness reduction of particular substructures of the bridge and are simulated by reducing the modulus of elasticity of these substructures. The first damage case (Figure 1a) corresponds to damage in the left-support elastomeric bearing simulated by reducing the stiffness of the bearing by $50 \%$ in the two horizontal directions, longitudinal $x$ and transverse $y$. The second damage case (Figure 1b) corresponds to damage in the top right section of the central pier which is simulated by reducing the modulus of elasticity of the top right pier by $50 \%$. Simulated modal data are generated from the finite element models of the undamaged and damaged structure. To simulate the effects of measurement noise and modeling error, 2\% and 5\% Gaussian noise are respectively added to the modal frequencies and modeshapes simulated by the finite element models. These simulated, noisy contaminated, modal data $\hat{\omega}_{r}$ and $\hat{\phi}_{r}$ are then used in the methodology to predict the location and severity of damage.

Following the proposed damage detection methodology, a family of 12 alternative model classes $\left\{M_{1}, \cdots, M_{12}\right\}$ is introduced to monitor different plausible damage scenarios. All competitive finite element model classes are generated from the nominal 1350-DOF finite element model and differ by the parameterization scheme. Each model class is parameterized by one or more stiffness-related parameters, shown in Table 1, accounting for the stiffness properties of various substructures of the bridge. The properties of the stiffness elements that are not parameterized in each model class are equal to the nominal values of the reference finite element model of the bridge in its undamaged state. It can be observed that one or more of the introduced model classes "contain" other model classes. For example, the model class $M_{3}$ "contains" the model classes $M_{1}$ and $M_{2}$ in the sense that the model class $M_{3}$ can predict the damage scenarios that can be predicted by the model classes $M_{1}$ and $M_{2}$. In particular, the model class $M_{12}$ contains all other model classes $M_{1}$ to $M_{11}$.

For each damage case introduced in Figure 1, Table 2 gives the results of the probability $P\left(M_{i} \mid D\right)$ of each model class, indicative of the location of damage, 
and the percentage change $\Delta \boldsymbol{\theta}_{i}$, indicative of the severity of damage. Results are presented for three cases corresponding to different number $m=10,6$ and 3 of contributing modes and three different number $N_{0}$ of sensors involving respectively $N_{0}=14,6$ and 3 sensors with locations and directions of sensors as shown in Figure 2.

Table 2 gives the results only for the model classes that contain or partially contain the damage. All other model classes are found to have zero probability and so results are not given in Table 2. For the first damage case it is expected that the methodology will give as the most probable model class one of the $M_{3}, M_{8}$, $M_{9} \kappa \alpha M_{12}$ that contain the damaged substructure. Comparing the probability of each model class and also the corresponding magnitude of damage predicted by each model class it is evident that the proposed methodology correctly predicts the location of damage, while the prediction of the magnitude of damage is considered satisfactory. Indeed the most probable model class is one of the $M_{3}$, $M_{8}, M_{12}$, depending on the number of modes and the number of sensors. From the $\Delta \boldsymbol{\theta}_{i}$ values predicted by the most probable model classes $M_{3}, M_{8} \kappa \alpha 1 M_{12}$, but also for the model class $M_{9}$ that contains the damaged substructure although it is not favored by the method, it results that the damage is concentrated in the left bearing along the $x$ and $y$ directions. Specifically, in the case of $m=10$ and $N_{0}=14$, the magnitude of damage is predicted correctly from the most probable model class $M_{8}$ with $P\left(M_{8} \mid D\right)=0.83$, to be approximately $52 \%$ along the $x$ direction and $49 \%$ along the $y$ direction. In the case of $m=6$ and $N_{0}=6$, the methodology predicts with equal probability the two model classes $M_{3}$ and $M_{8}$ that contain the damage with probabilities $P\left(M_{3} \mid D\right)=P\left(M_{8} \mid D\right)=0.49$. For any of these model classes, the magnitude of damage is correctly predicted to be approximately $50 \%$ to $51 \%$ in the left bearing along the $x$ and $y$ directions, respectively. In the case $m=3$ and $N_{0}=3$, the method favors the model class $\mathrm{M}_{12}$ that correctly predicts with $P\left(M_{12} \mid D\right)=1$ the location of damage to be in the left bearing, but it overestimates a severity of $68 \%$ damage in the $x$ direction, and it also predicts significant damage of the order of $75 \%$ in the central segment of the pier. The failure of the methodology to give accurate results for the location and the magnitude of damage is due to the inadequate information contained in 
the 3 lowest modal characteristics $(m=3$ ) for the particular configuration of only three sensors $\left(N_{0}=3\right)$.

For the second damage case, it is expected that the methodology will give as the most probable model class one of the $M_{10}$ and $M_{12}$ that contain the damaged substructure. Results are also presented for the model class $M_{11}$ which partially contains the damage in the sense that damage in the top right section of the pier can be partially monitored by this model class. From Table 2 results, it is observed in this damage case that the methodology correctly predicts the general area of damage to be at the pier, but it fails to identify exactly which of the three sections of the pier is damaged. Indeed, comparing the probability of each model class, it is evident that the most probable model class is one of $M_{11}$ and $M_{12}$ depending on the number $m$ of models and the number $N_{0}$ of sensors. In the case of $m=10$ and $N_{0}=14$, the methodology favors $M_{11}$ that correctly predicts only the general area of damage to be at the pier, but it is unable to predict the particular section that contains the damage. Specifically, the inflicted damage of $50 \%$ in the right to section of the pier is shared by the three sections in an amount of $22 \%$. Also, the model classes $M_{10}$ and $M_{12}$ that fully contain the damaged substructure, fail to accurately predict the actual magnitude of damage, sharing the damage among the three section of the pier. In the case $m=6$ and $N_{0}=6$, the methodology favors $\mathrm{M}_{12}$ with probability $P\left(M_{12} \mid D\right)=0.8$ that correctly predicts the location of damage in the right top section of the pier. Also, it predicts with satisfactory accuracy the magnitude of damage to be $54 \%$ instead of the inflicted $50 \%$. In the case $m=3$ and $N_{0}=3$ of small number of data, the methodology also favors $M_{12}$ that correctly predicts the general area of damage, but the magnitude of damage is shared among the 3 sections of the pier, 23\% in the right top section, 38\% in the left top section and $38 \%$ in the central section of the pier. The failure of the methodology to accurately detect the section that is damaged is partly due to the fact that the measurements do not contain enough spatially-distributed information for distinguishing and localizing damage within the three sections of the pier, and partly due to inadequate information contained in the measurements with small number of sensors and limited number of contributing modes. A more effective localization of damage in one of the three sections can be achieved only 
if measurements are obtained from sensors that are located along the height of the pier.

For given size of model and measurement error, the effectiveness of the methodology in predicting the location and magnitude of damage depends on the number of contributing modes, as well as the number and location of sensors. It should be pointed out, however, that in the absence of modeling and measurements errors, the proposed methodology using model updating tools capable of finding the global optimal structural models (Christodoulou and Papadimitriou 2007), can reliably predict the exact locations and provide accurate estimates of the magnitude of damages, provided that one of the model classes contains the damaged substructure.

\subsection{Damage detection for a small-scaled laboratory bridge section}

The methodology is next validated using measured modal data from a laboratory small-scaled section of a bridge shown in Figure 3a. The laboratory structure is made of steel and simulates a simply supported section of a bridge resting on rigid foundation through bearings. In order to avoid nonlinear phenomena due to sliding of the bearings during the vibration of the bridge, the faces of the bearings are glued to the foundation and the bridge deck. The bearings are simulated using square sections of White Nylon 66 material of edge size $14 \mathrm{~mm}$. Damage is simulated at the bearings by changing the size of the left and right bearings. This change is achieved by replacing the bearings with smaller ones of edge size $10 \mathrm{~mm}$.

The section of the beam at its undamaged and its damaged state was instrumented with 14 accelerometers, measuring along the longitudinal (2 sensors), vertical ( 8 sensors) and transverse ( 4 sensors) directions. The modal characteristics of the undamaged and damaged structure were obtained by analysing measured acceleration response time histories from several impulse hammer tests using conventional modal analysis software that processes simultaneously the transfer functions at the measured locations. The damage detection methodology make use of the following five modal frequencies and modeshapes of the undamaged and damaged structure: $1^{\text {st }}$ longitudinal, $1^{\text {st }}$ and $2^{\text {nd }}$ bending, $1^{\text {st }}$ transverse and $1^{\text {st }}$ torsional. The corresponding identified values of the modal frequencies are (in Hz): 108.7, 18.52, 60.08, 31.10 and 46.65 for the 
undamaged structure, and 69.74, 17.08, 59.22, 29.98 and 42.96 for the damaged one.

A finite element model was also constructed using beam elements to describe the behaviour of the bridge in its undamaged and damaged states. The deck and the bearings were modeled using three-dimensional two-node elements. The total number of DOF is 350 . The finite element model was first calibrated to fit the modal characteristics of the undamaged structure using the model updating methodologies presented in the companion paper (Ntotsios et al. 2008). The modal characteristics of the damaged structure which contain significant information about the damaged state at the bearings were then used to predict the damage location and severity based on the proposed damage detection methodology.

Based on the damaged detection methodology, nine (9) competitive model classes $\left\{M_{1}, \cdots, M_{9}\right\}$, given in Table 3 , were introduced to monitor various probable damage scenarios corresponding to single and multiple damages at different substructures. The stiffness related parameters used in each model class involve the modulus of elasticity $E$ of the deck, the modulus of elasticity $E$ of the bearings and the cross-sectional moment of inertia $I_{x x}$ and $I_{y y}$ of the bearings with respect to the global coordinate system shown in Figure 3b. All model classes are generated from the updated finite element model of the undamaged structure. Based on the parameterization shown in Table 3, it is expected that the methodology will give as the most probable model class one of $M_{4}, M_{5}, M_{7}$ and $M_{9}$ that contain the actual damage. The results for the probability of each model class and the value of the measure of fit $J_{i} \equiv J\left(\theta_{i} ; M_{i}, D\right)$, given in (6), between the measured and the optimal model predicted modal characteristics for all model classes, are also reported in Table 3.

Comparing the probability $P\left(M_{i}\right)$ of each model class and also the corresponding magnitude of damages $\Delta \boldsymbol{\theta}_{i}$ predicted by each model class it is evident that the proposed methodology correctly predicts the location and magnitude of damage. Among all alternative model classes $M_{4}, M_{5}, M_{7}$ and $M_{9}$ that contain the actual damage, although the model classes $M_{5}$ and $M_{9}$ predict the smallest measure of fit $J$, the proposed methodology favors with probability 0.708 the model class $M_{4}$ with the least number of parameters, which is 
consistent with theoretical results available for Bayesian model class selection (Beck and Yuen 2004). The reduction of 55.2\% of the modulus of elasticity at the left and right bearings, predicted by the most probable model class $M_{4}$, is an indication of the severity of damage caused by reducing the edge length of the bearings from $14 \mathrm{~mm}$ to $10 \mathrm{~mm}$. The model class $M_{5}$ is the second most probable model class, involving two parameters, favored with probability $P\left(M_{5} \mid D\right)=0.239$ and correctly predicts the magnitude of damage to be $54.3 \%$, at approximately the same level as that predicted by the most probable model class $M_{4}$. The third most probable model class $M_{2}$, although it does not contained the damage, it is favored by the methodology in relation to the other two model classes $M_{7}$ and $M_{9}$ that contain the damage. The model class $M_{2}$ predicts damage of magnitude $68.4 \%$ at the left bearing, while by construction it fails to predict damage at the right bearing since there are no parameters in this model class to monitor changes in the right bearings. The model classes $M_{7}$ and $M_{9}$, involving two and three parameters, respectively, also correctly predict the magnitude of damage to be at the same levels (approximately 59\% at the left bearing and $49 \%$ to $52 \%$ at the right bearing) as that predicted by the most probable model class $M_{4}$. The slight differences in the predictions from the model classes that contain the damage and the slight increase of the stiffness for the deck, of the order of $7 \%$, predicted from model classes $M_{5}$ and $M_{9}$ are due to the measurement and model errors.

\section{Conclusions}

A bridge health monitoring system using vibration measurements was outlined in this work. In particular, a Bayesian inference methodology was presented for the identification of the location and the severity of damage using measured modal characteristics. The effectiveness of the damage detection methodology was illustrated using simulated modal data from the Polymylos bridge of the Egnatia Odos motorway (Greece) and measured data from a small scaled laboratory section of a bridge. Results provided useful information on the strength and limitations of the methodology. Specifically, the effectiveness of the methodology depends on several factors, including 
- Model classes and parameterization (number and type of parameters) that are introduced to simulate the possible damage scenarios. At least one member in the family of model classes should contain, partially or fully, the actual damage scenario, otherwise the damage prediction from the methodology is ineffective.

- Type, location and magnitude of damage or damages in relation to the sensor network configuration (number and location of sensors). Measurements should contain adequate information for simultaneously identifying all model classes introduced for monitoring possible damage scenarios, as well as estimating their parameter values.

- Model and measurement errors in relation to the magnitude of damage. Damages of small magnitude in relation to model error and measurement noise may be hidden and difficult to be identified. Damage predictions can be improved by introducing high fidelity finite element model classes and estimation algorithms that provide more accurate values of the modal characteristics.

The proposed framework can be used by highway managing authorities as part of an intelligent bridge management system to provide a useful tool for the continuous monitoring of bridges and assessment of structural integrity.

Acknowledgements: This research was partially funded by the General Secretariat of Research and Technology of Greece and the European Union (European Social Fund), through the EPAN program ASProGe (Seismic Protection of Bridges) under grant DP15. Also, this research was partially funded by the Greek National Scholarships Foundation (IKY). Both supports are gratefully acknowledged.

\section{References}

Au SK (2001) On the Solution of the First Excursion Problem by Simulation with Applications to Probabilistic Seismic Performance Assessment. Ph. D. thesis, EERL Report 2001-02, Caltech, Pasadena, CA.

Beck JL, and Au SK (2002) Bayesian Updating of Structural Models and Reliability using Markov Chain Monte Carlo Simulation. Journal of Engineering Mechanics (ASCE), 128 (4): 380-391.

Beck JL, Katafygiotis LS (1998) Updating models and their uncertainties. I: Bayesian statistical framework. Journal of Engineering Mechanics (ASCE) 124 (4): 455-461.

Beck JL, Yuen KV (2004) Model selection using response measurements: Bayesian probabilistic approach. Journal of Engineering Mechanics (ASCE) 130(2):192-203. 
Christodoulou K, Papadimitriou C (2007) Structural identification based on optimally weighted modal residuals. Mechanical Systems and Signal Processing 21: 4-23.

COMSOL AB (2005) COMSOL Multiphysics User's Guide. [http://www.comsol.com/].

Doebling S, Farrar C, Prime M, and Shevitz D (1998) Damage Identification and Health Monitoring of Structural and Mechanical Systems from Changes in their Vibration Characteristics: A Literature Review. Report LA-13070-MS, Los Alamos National Laboratory.

Fritzen CP, Jennewein D, Kiefer T (1998) Damage detection based on model updating methods. Mechanical Systems and Signal Processing 12(1): 163-186.

Goursat M, Basseville M, Benveniste A, Mevel L (2000) A Scilab toolbox for output only modal analysis and diagnosis. Proceedings 18th International Modal Analysis Conference, San Antonio, Texas. [ftp://ftp.inria.fr/INRIA/Projects/Meta2/Scilab/contrib/MODAL/].

Hemez FM, and Farhat C (1995) Structural Damage Detection via a Finite Element Model Updating Methodology. The International Journal of Analytical and Experimental Modal Analysis 10 (3): 152-166.

Katafygiotis LS, and Cheung SH (2002) MCMC Based Simulation Methodology for Reliability Calculations. Fourth International Conference on Computational Stochastic Mechanics, Spanos PD, and Deodatis G, (Eds). Millpress, Rotterdam, the Netherlands 293-299.

Katafygiotis LS, Papadimitriou C, Lam HF (1998) A probabilistic approach to structural model updating. International Journal of Soil Dynamics and Earthquake Engineering 17 (7-8): 495 507.

Kirkegaard PH, Brincker R (1994) On the optimal locations of sensors for parametric identification of linear structural systems. Mech Syst Signal Process 8: 639-647.

Lam HF, Ng CT, Veidt M (2007) Experimental characterization of multiple cracks in a cantilever beam utilizing transient vibration data following a probabilistic approach. Journal of Sound and Vibration, 305(1-2): 34-49.

Lam HF, Katafygiotis LS, Mickleborough NC (2004) Application of a statistical model updating approach on phase I of the IASC-ASCE structural health monitoring benchmark study. Journal of Engineering Mechanics (ASCE) 130 (1): 34-48.

Mottershead JE, Friswell MI (1993) Model updating in structural dynamics: A survey. Journal of Sound and Vibration 167 (2): 347-375.

Ntotsios E, Karakostas Ch, Lekidis V, Panetsos P, Nikolaou I, Papadimitriou C (2008). Structural identification of Egnatia Odos bridges using ambient and earthquake-induced vibrations. Bulleting on Earthquake Engineering, under review.

Papadimitriou C (2005) Pareto Optimal Sensor Locations for Structural Identification, Computer Methods in Applied Mechanics and Engineering, 194(12-16): 1655-1673.

Papadimitriou C (2004) Bayesian inference applied to structural model updating and damage detection. 9th ASCE Joint Specialty Conference on Probabilistic Mechanics and Structural Reliability, Albuquerque, New Mexico.

Papadimitriou C, Katafygiotis LS (2004) Bayesian modeling and updating. In Engineering Design Reliability Handbook, Nikolaidis N, Ghiocel DM, Singhal S (Eds), CRC Press. 
Papadimitriou C, Beck JL, Katafygiotis LS (2001) Updating robust reliability using structural test data. Probabilistic Engineering Mechanics, 16(2): 103-113.

Papadimitriou C, Beck JL, Katafygiotis LS (1997) Asymptotic Expansions for Reliability and Moments of Uncertain Systems. Journal of Engineering Mechanics (ASCE), 123(12): 12191229.

Peeters B, Van Den Branden B, Laquiere A, De Roeck G (1999) Output-only modal analysis: development of a GUI for Matlab. In Proceedings of IMAC 17, Kissimmee, FL, USA, 10491055.

Reynders E, De Roeck G (2007) What's new in system identification for experimental and operational modal analysis. ECCOMAS Thematic Conference on Computational Methods in Structural Dynamics and Earthquake Engineering, Papadrakakis M, Charmpis DC, Lagaros ND, Tsompanakis Y (eds.) Rethymno, Crete, Greece, 13-16 June 2007.

Sohn H, Law KH (1997) Bayesian probabilistic approach for structural damage detection. Earthquake Engineering and Structural Dynamics 26: 1259-1281.

Teughels A, De Roeck G (2005) Damage detection and parameter identification by finite element model updating. Archives of Computational Methods in Engineering 12(2): 123-164,.

Vanik MW, Beck JL, Au SK (2000) Bayesian probabilistic approach to structural health monitoring. Journal of Engineering Mechanics (ASCE) 126 (7): 738-745.

Verboven P, Cauberghe B, Parloo E, Vanlanduit S, Guillaume P (2004) User-assisting tools for a fast frequency-domain modal parameter estimation method. Mechanical Systems and Signal Processing 18(4): 759-780.

Yuen KV (2002) Model selection identification and robust control for dynamical systems. Ph.D. Thesis, EERL Report 2002-03, Caltech, Pasadena. 


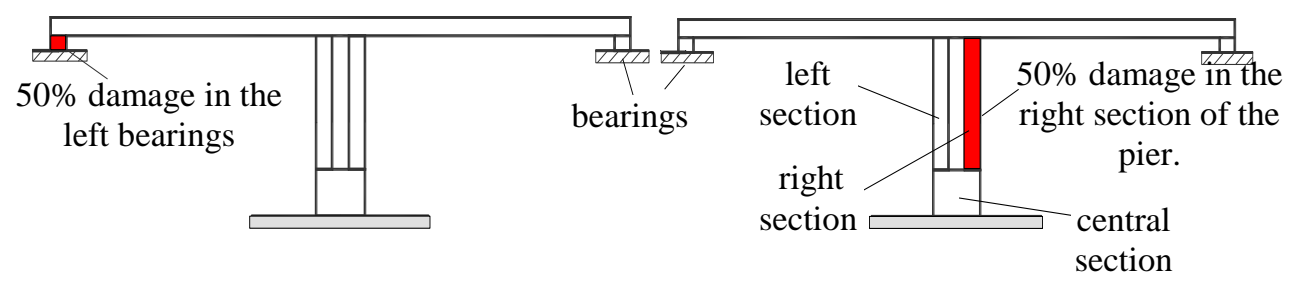

(a)

(b)

Figure 1: (a) First damage case at left bearing, (b) Second damage case in the top right section of the pier 


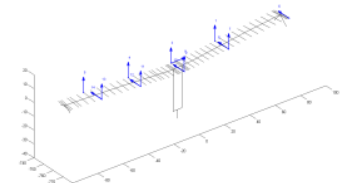

(a)

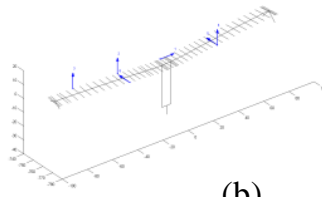

(b)

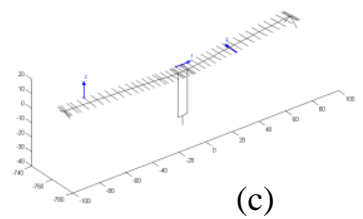

(c)

Figure 2: Sensor configurations for (a) 14 sensors, (b) 6 sensors and (c) 3 sensors. 


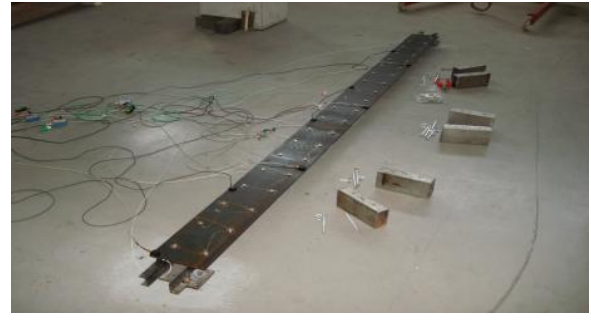

(a)

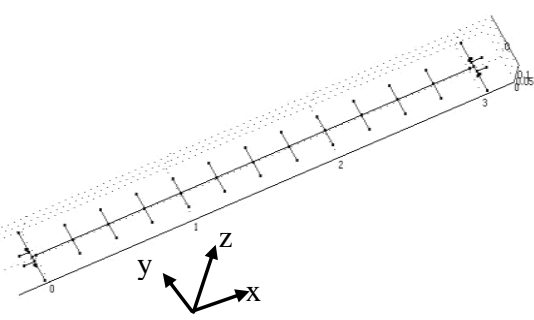

(b)

Figure 3: (a) Small scale section of bridge with sensors, (b) Finite element model. 
Table 1: Family of model classes $M_{i}, i=1, \cdots, \mu$, with parameterization

\begin{tabular}{|l|l|l|l|l|l|l|l|l|}
\hline & $\begin{array}{l}\text { Left } \\
\text { Bearing } \\
\text { Long. }\end{array}$ & $\begin{array}{l}\text { Left } \\
\text { Bearing } \\
\text { Trans. }\end{array}$ & $\begin{array}{l}\text { Right } \\
\text { Bearing } \\
\text { Long. }\end{array}$ & $\begin{array}{l}\text { Right } \\
\text { Bearing } \\
\text { Trans. }\end{array}$ & Deck & $\begin{array}{l}\text { Top } \\
\text { Right } \\
\text { Pier }\end{array}$ & $\begin{array}{l}\text { Top } \\
\text { Left } \\
\text { Pier }\end{array}$ & $\begin{array}{l}\text { Central } \\
\text { Pier }\end{array}$ \\
\hline$M_{1}$ & $\theta_{1}$ & & & & & & & \\
\hline$M_{2}$ & & $\theta_{1}$ & & & & & & \\
\hline$M_{3}$ & $\theta_{1}$ & $\theta_{2}$ & & & & & & \\
\hline$M_{4}$ & & & $\theta_{1}$ & & & & & \\
\hline$M_{5}$ & & & & $\theta_{2}$ & & & & \\
\hline$M_{6}$ & & & $\theta_{1}$ & $\theta_{2}$ & & & & \\
\hline$M_{7}$ & & & & & $\theta_{1}$ & & & \\
\hline$M_{8}$ & $\theta_{1}$ & $\theta_{2}$ & $\theta_{3}$ & $\theta_{4}$ & & & & \\
\hline$M_{9}$ & $\theta_{1}$ & $\theta_{1}$ & $\theta_{2}$ & $\theta_{2}$ & & & & \\
\hline$M_{10}$ & & & & & & $\theta_{1}$ & $\theta_{2}$ & $\theta_{3}$ \\
\hline$M_{11}$ & & & & & & $\theta_{1}$ & $\theta_{1}$ & $\theta_{1}$ \\
\hline$M_{12}$ & $\theta_{1}$ & $\theta_{2}$ & $\theta_{3}$ & $\theta_{4}$ & $\theta_{5}$ & $\theta_{6}$ & $\theta_{7}$ & $\theta_{8}$ \\
\hline
\end{tabular}

Long=Longitudinal direction, Trans=Transverse direction 
Table 2: Damage detection results for the two damage scenarios

\begin{tabular}{|c|c|c|c|c|c|c|}
\hline \multirow{4}{*}{$\begin{array}{l}\text { Model } \\
\text { Class }\end{array}$} & \multicolumn{3}{|c|}{ First Damage Scenario } & \multicolumn{3}{|c|}{ Second Damage Scenario } \\
\hline & $m=10$ & $m=6$ & $m=3$ & $m=10$ & $m=6$ & $m=3$ \\
\hline & $N_{0}=14$ & $N_{0}=6$ & $N_{0}=3$ & $N_{0}=14$ & $N_{0}=6$ & $N_{0}=3$ \\
\hline & \multicolumn{6}{|c|}{ Probability $P\left(M_{i}\right)$ of each model class } \\
\hline$M_{3}$ & 0.12 & 0.49 & 0 & - & - & - \\
\hline$M_{8}$ & 0.83 & 0.49 & 0 & - & - & - \\
\hline$M_{9}$ & 0 & 0 & 0 & - & - & - \\
\hline$M_{10}$ & - & - & - & 0 & 0.2 & 0 \\
\hline$M_{11}$ & - & - & - & 1 & 0 & 0 \\
\hline$M_{12}$ & 0 & 0 & 1 & 0 & 0.8 & 1 \\
\hline \multicolumn{7}{|c|}{ Predicted Magnitude of Damage $\Delta \boldsymbol{\theta}_{1}-\Delta \boldsymbol{\theta}_{2}-\cdots-\Delta \boldsymbol{\theta}_{\mu}(\%)$} \\
\hline$M_{3}$ & $53-50$ & $51-50$ & $50-50$ & - & - & - \\
\hline$M_{8}$ & $52-49-0-0$ & $51-50-0-0$ & $59-50-0-13$ & - & - & - \\
\hline$M_{9}$ & $49-0$ & $50-0$ & $50-1$ & - & - & - \\
\hline$M_{10}$ & - & - & - & $33-13-6$ & $54-0-0$ & $33-17-24$ \\
\hline$M_{11}$ & - & - & - & 22 & 21 & 25 \\
\hline$M_{12}$ & $\begin{array}{l}52-49-0-0- \\
0-0-0-0\end{array}$ & $\begin{array}{l}51-49-3-0- \\
0-0-0-7\end{array}$ & $\begin{array}{l}68-48-0-0- \\
0-0-0-75\end{array}$ & $\begin{array}{l}0-0-0-0-0 \\
-31-10-22 \\
\end{array}$ & $\begin{array}{l}0-0-0-0-0- \\
54-0-7\end{array}$ & $\begin{array}{l}4-0-0-7-0- \\
23-38-38\end{array}$ \\
\hline
\end{tabular}


Table 3: Probability $P\left(M_{i}\right)$ of each model class and predicted magnitude of damage $\Delta \boldsymbol{\theta}$

\begin{tabular}{|c|c|c|c|c|c|}
\hline $\begin{array}{c}\text { Model } \\
\text { Class }\end{array}$ & $N_{\theta_{i}}$ & Prob. $P\left(M_{i}\right)$ & Fit $J_{i}$ & Parameters & $\begin{array}{c}\text { Damage } \Delta \theta \\
(\%)\end{array}$ \\
\hline$M_{1}$ & 1 & 0 & 0.1444 & $E$ deck & -14.1 \\
\hline$M_{2}$ & 1 & 0.029 & 0.0223 & $E$ left bearing & -68.4 \\
\hline$M_{3}$ & 1 & 0.003 & 0.0241 & $E$ right bearing & -67.7 \\
\hline$M_{4}$ & 1 & 0.708 & 0.0202 & $\begin{array}{c}E \text { left \& right } \\
\text { bearings }\end{array}$ & -55.2 \\
\hline \multirow{2}{*}{$M_{5}$} & \multirow[b]{2}{*}{2} & \multirow[b]{2}{*}{0.239} & \multirow[b]{2}{*}{0.0184} & $E$ deck & +6.93 \\
\hline & & & & $\begin{array}{c}E \text { left \& right } \\
\text { bearings }\end{array}$ & -54.3 \\
\hline \multirow{2}{*}{$M_{6}$} & \multirow{2}{*}{2} & \multirow{2}{*}{0.000} & \multirow{2}{*}{0.0299} & $I_{x x}$ bearings & -52.1 \\
\hline & & & & $I_{y y}$ bearings & -53.3 \\
\hline \multirow{2}{*}{$M_{7}$} & \multirow{2}{*}{2} & \multirow{2}{*}{0.014} & \multirow{2}{*}{0.0201} & $E$ left bearings & -58.4 \\
\hline & & & & $E$ right bearings & -51.8 \\
\hline \multirow{3}{*}{$M_{8}$} & \multirow{3}{*}{3} & \multirow{3}{*}{0} & \multirow{3}{*}{0.0280} & $E$ deck & -3.31 \\
\hline & & & & $I_{x x}$ bearings & -52.6 \\
\hline & & & & $I_{y y}$ bearings & -54.2 \\
\hline \multirow{3}{*}{$M_{9}$} & \multirow{3}{*}{3} & \multirow{3}{*}{0.007} & \multirow{3}{*}{0.0180} & $E$ deck & +7.13 \\
\hline & & & & $E$ left bearings & -58.9 \\
\hline & & & & $E$ right bearings & -49.3 \\
\hline
\end{tabular}

